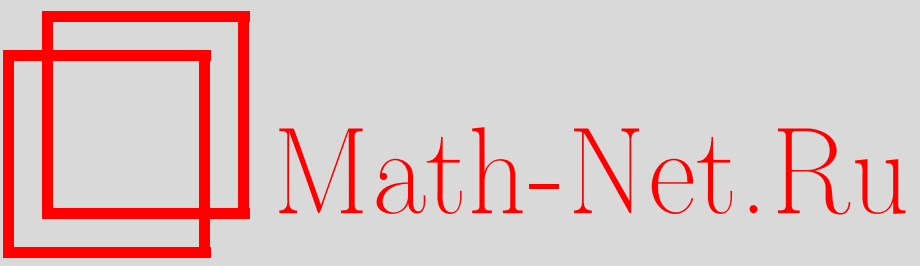

С. В. Галаев, О геодезических преобразованиях распределений субримановых многообразий, Итоги науки и техн. Сер. Соврем. мат. и ее прил. Темат. обз., 2020, том 182, 14-18

DOI: https://doi.org/10.36535/0233-6723-2020-182-14-18

Использование Общероссийского математического портала Math-Net.Ru подразумевает, что вы прочитали и согласны с пользовательским соглашением

http: //www. mathnet.ru/rus/agreement

Параметры загрузки:

IP: 52.23 .180 .231

26 апреля 2023 г., 12:11:44 


\title{
О ГЕОДЕЗИЧЕСКИХ ПРЕОБРАЗОВАНИЯХ РАСПРЕДЕЛЕНИЙ СУБРИМАНОВЫХ МНОГООБРАЗИЙ
}

\author{
(c) 2020 г. \\ С. В. ГАЛАЕВ
}

\begin{abstract}
АннотАция. Пусть $M$ - субриманово многообразие контактного типа с распределением $D$. C помощью эндоморфизма $N: D \rightarrow D$ распределения $D$ внутренняя связность, осуществляющая перенос допустимых векторов вдоль допустимых кривых многообразия $M$, продолжается до связности в векторном расслоении $(D, \pi, M)$, где $\pi: D \rightarrow M$-естественная проекция. Полученная связность названа в работе $N$-продолженной связностью. Задание $N$-продолженной связности эквивалентно заданию на распределении $D N$-продолженной субримановой структуры. С помощью структурных уравнений $N$-продолженной структуры вычислены коэффициенты связности ЛевиЧивиты, полученной в результате продолжения риманова многообразия. Доказано, что если распределение $D$ субриманова многообразия не интегрируемо, то две $N$-продолженные субримановы структуры контактного типа, одна из которых определяется нулевым эндоморфизмом, а другая произвольным ненулевым эндоморфизмом, принадлежат разным геодезическим классам.
\end{abstract}

Ключевые слова: субриманово многообразие контактного типа, $N$-продолженная связность, геодезическое преобразование.

\section{GEODESIC TRANSFORMATIONS OF DISTRIBUTIONS OF SUB-RIEMANNIAN MANIFOLDS}

\author{
(c) 2020 S. V. GALAEV
}

\begin{abstract}
Let $M$ be a sub-Riemannian contact-type manifold endowed with a distribution $D$. Using an endomorphism $N: D \rightarrow D$ of the distribution $D$, one can prolong the inner connection, which transfers admissible vectors along admissible curves on the manifold $M$, up to a connection in the vector bundle $(D, \pi, M)$, where $\pi: D \rightarrow M$ is the natural projection. The connection obtained is called the $N$-prolonged connection. The setting of an $N$-prolonged connection is equivalent to the setting of an $N$-prolonged sub-Riemannian on the distribution $D$. Using the structure equations of the $N$-prolonged structure, we calculate the coefficients of the Levi-Civita connection obtained by the prolongation of the Riemannian manifold. We prove that if a distribution $D$ of a sub-Riemannian manifold is not integrable, then two $N$-prolonged, contact-type, sub-Riemannian structures, one of which is determined by the zero endomorphism and the other by an arbitrary nonzero endomorphism, belong to distinct geodesic classes.
\end{abstract}

Keywords and phrases: sub-Riemannian manifold of contact type, $N$-extended connection, geodesic transformation.

AMS Subject Classification: 53C17

1. Введение. Систематическое изучение геометрии распределений почти контактных метрических многообразий, а затем и распределений субримановых многообразий начинается в $[4,7,8,12]$. Как показано в [4], распределение субриманова многообразия может быть использовано для моделирования неголономных механических систем. На распределении субриманова 
многообразия естественным образом возникает контактное векторное поле - аналог гамильтоновой системы. В [4] излагаются методы, позволяющие находить первые интегралы обобщенной гамильтоновой системы (контактного векторного поля). Полученные в [4] результаты основаны на исследовании структур, определяемых на распределениях субримановых многообразий. В настоящей работе эти структуры получили название $N$-продолженных структур. Как и исходные структуры, продолженные структуры являются субримановыми структурами. Распределения субримановых многообразий допускает задание более богатых структур (см. $[1,2,10])$, чем субримановы структуры контактного типа, но их изучение избыточно по отношению к решаемой в этой статье задаче.

Пусть $(M, \boldsymbol{\xi}, \eta, g, D)$ - субриманова структура контактного типа (см. [5]). Для задания $N$-продолженной структуры используется такой эндоморфизм $N: T M \rightarrow T M$, что для всех $x \in M$, для которых $N\left(D_{x}\right) \subset D_{x}$, выполняется равенство $N \boldsymbol{\xi}=0$. Наличие условия $N \boldsymbol{\xi}=0$ означает, что эндоморфизм $N$ является допустимым эндоморфизмом (см. $[7,12])$, поэтому в дальнейшем будем писать $N: D \rightarrow D$. Задание эндоморфизма $N$ позволяет продолжить внутреннюю связность до связности в векторном расслоении $(D, \pi, M)$, где $\pi: D \rightarrow M$-естественная проекция.

Идея построения $N$-связностей берет начало в работе В. В. Вагнера, посвященной развитию геометрической теории неголономной механики (см. [6]). С геометрической точки зрения уравнения движения неголономной динамической системы интерпретируются как уравнения геодезических внутренней связности (см. $[7,12])$, заданной на неголономном многообразии - конфигурационном пространстве механической системы. В некоторых случаях геометрические свойства неголономного многообразия позволяют выбрать такую специальную систему координат, в которой уравнения движения неголономной динамической системы принимают наиболее простой вид. Поиск необходимых геометрических инвариантов привел В. В. Вагнера к построению тензора кривизны неголономного многообразия, называемого в настоящее время тензором кривизны Вагнера. В [9] автором статьи было показано, что в случае контактного многообразия тензор кривизны Вагнера совпадает с тензором кривизны некоторой связности в векторном расслоении $(D, \pi, M)$, пространством которого является распределение $D$ контактной структуры $(M, \boldsymbol{\xi}, \eta, \varphi)$. Необходимая для получения тензора кривизны Вагнера связность была названа связностью Вагнера. Задание связности Вагнера сводится к продолжению внутренней связности до связности в векторном расслоении с помощью эндоморфизма $N: D \rightarrow D$, имеющего специальное строение.

Пусть $\left(D, \partial_{n}, \lambda=\eta \circ \pi_{*}, G, \tilde{D}\right),\left(D, \boldsymbol{u}, \lambda=\eta \circ \pi_{*}, \tilde{G}, \tilde{D}\right)$ - две $N$-продолженные структуры контактного типа. Причем первая структура получена с помощью нулевого эндоморфизма, вторая с помощью произвольного ненулевого эндоморфизма $N: D \rightarrow D$. В работе показано, что соответствующие римановы многообразия $(D, G),(D, \tilde{G})$ принадлежат разным геодезическим классам.

2. $\quad N$-продолженная связность на субримановом многообразии. Пусть $M$ - субриманово многообразие контактного типа, т.е. гладкое многообразие размерности $n$ с заданной на нем субримановой структурой $(M, \boldsymbol{\xi}, \eta, g, D)$, где $\eta-1$-форма, порождающая распределение $D: D=$ $\operatorname{ker}(\eta) ; \boldsymbol{\xi}$ - векторное поле, порождающее оснащение $D^{\perp}$ распределения $D: D=\operatorname{Span}(\boldsymbol{\xi}) ; g-$ риманова метрика на многообразии $M$, относительно которой распределения $D$ и $D^{\perp}$ взаимно ортогональны. При этом выполняются равенства $\eta(\boldsymbol{\xi})=1$ и $g(\boldsymbol{\xi}, \boldsymbol{\xi})=1$.

Назовем распределение $D$ распределением субримановой структуры. Для проведения необходимых вычислений будем использовать атлас карт $K\left(x^{\alpha}\right)(\alpha, \beta, \gamma=1, \ldots, n ; a, b, c=1, \ldots, n-1$; $i, j, k=1, \ldots, 2 n-1)$, удовлетворяющих условию $\partial_{n}=\boldsymbol{\xi}$ (см. [3]). Пусть $P: T M \rightarrow D$-оператор проектирования, определяемый разложением $T M=D \oplus D^{\perp}$. Линейно независимые в каждой точке векторные поля $P\left(\partial_{a}\right)=\boldsymbol{e}_{a}=\partial_{a}-\Gamma_{a}^{n} \partial_{n}$ порождают систему $D: D=\operatorname{Span}\left(\boldsymbol{e}_{a}\right)$. Легко проверить, что $\left[\boldsymbol{e}_{a}, \boldsymbol{e}_{b}\right]=2 \omega_{b a} \partial_{n}$.

Рассмотрим допустимые тензорные поля $($ см. $[7,12])$ следующего вида:

$$
h \boldsymbol{x}=\frac{1}{2}\left(L_{\boldsymbol{\xi}} \varphi\right)(\boldsymbol{x}), \quad C(\boldsymbol{x}, \boldsymbol{y})=\frac{1}{2}\left(L_{\boldsymbol{\xi}} g\right)(\boldsymbol{x}, \boldsymbol{y}), \quad g(C \boldsymbol{x}, \boldsymbol{y})=C(\boldsymbol{x}, \boldsymbol{y}), \quad \boldsymbol{x}, \boldsymbol{y} \in \Gamma(T M) .
$$


В адаптированных координатах имеем

$$
h_{b}^{a}=\frac{1}{2} \partial_{n} \varphi_{b}^{a}, \quad C_{a b}=\frac{1}{2} \partial_{n} g_{a b}, \quad C_{b}^{a}=g^{d a} C_{d b}, \quad \varphi_{a}^{c}=g^{b c} \omega_{b a} .
$$

Обозначим коэффициенты связности Леви-Чивиты тензора $g$ символами $\Gamma_{\beta \gamma}^{\alpha}$. Имеет место следующее предложение (см. [9]).

Предложение 1. Коэффициенты связности Леви-Чивиты контактного метрического многообразия в адаптированных координатах имеют вид

$$
\Gamma_{b c}^{a}=\frac{1}{2} g^{a d}\left(\boldsymbol{e}_{b} g_{c d}+\boldsymbol{e}_{c} g_{b d}-\boldsymbol{e}_{d} g_{b c}\right), \quad \Gamma_{a b}^{n}=\omega_{b a}-C_{a b}, \quad \Gamma_{a n}^{b}=\Gamma_{n a}^{b}=C_{a}^{b}-\varphi_{a}^{b}, \quad \Gamma_{n a}^{n}=\Gamma_{n n}^{a}=0 .
$$

Под внутренней линейной связностью на многообразии с контактной метрической структурой (см. $[3,9])$ будем понимать отображение $\nabla: \Gamma(D) \times \Gamma(D) \rightarrow \Gamma(D)$, обладающее следующими свойствами:

(1) $\nabla_{f_{1} \boldsymbol{x}+f_{2} \boldsymbol{y}}=f_{1} \nabla_{\boldsymbol{x}}+f_{2} \nabla_{\boldsymbol{y}}$

(2) $\nabla_{\boldsymbol{x}} f \boldsymbol{y}=(\boldsymbol{x} f) \boldsymbol{y}+f \nabla_{\boldsymbol{x}} \boldsymbol{y}$

(3) $\nabla_{\boldsymbol{x}}(\boldsymbol{y}+\boldsymbol{z})=\nabla_{\boldsymbol{x}} \boldsymbol{y}+\nabla_{\boldsymbol{x}} \boldsymbol{z}$

где $\Gamma(D)$ - модуль допустимых векторных полей. Коэффициенты линейной связности задаются соотношениями $\nabla_{\boldsymbol{e}_{a}} \boldsymbol{e}_{b}=\Gamma_{a b}^{c} \boldsymbol{e}_{c}$. Формулы преобразования для коэффициентов связности имеют обычный вид:

$$
\Gamma_{a b}^{c}=A_{a}^{a^{\prime}} A_{b}^{b^{\prime}} A_{c^{\prime}}^{c} \Gamma_{a^{\prime} b^{\prime}}^{c^{\prime}}+A_{c^{\prime}}^{c} e_{a} A_{b}^{c^{\prime}} .
$$

Кручение внутренней линейной связности $S$ задается равенством

$$
S(\boldsymbol{x}, \boldsymbol{y})=\nabla_{\boldsymbol{x}} \boldsymbol{y}-\nabla_{\boldsymbol{y}} \boldsymbol{x}-P[\boldsymbol{x}, \boldsymbol{y}] .
$$

В адаптированных координатах кручение внутренней связности получает следующее представление:

$$
S_{a b}^{c}=\Gamma_{a b}^{c}-\Gamma_{b a}^{c} .
$$

Говорят, что над распределением $D$ задана связность, если распределение $\tilde{D}=\pi_{*}^{-1}(D)$, где $\pi: D \rightarrow M$ - естественная проекция, раскладывается в прямую сумму вида $\tilde{D}=H D \oplus V D$, где $V D$ - вертикальное распределение на тотальном пространстве распределения $D$.

Гладкая структура на распределении $D$ задается следующим образом. Каждой адаптированной карте $K\left(x^{\alpha}\right)$ многообразия $M$ ставится в соответствие сверхкарта $\tilde{K}\left(x^{\alpha}, x^{n+a}\right)$ на многообразии $D$, где $x^{n+a}$ - координаты допустимого вектора в базисе $\boldsymbol{e}_{a}=\partial_{a}-\Gamma_{a}^{n} \partial_{n}$. Задание связности над распределением эквивалентно заданию такого объекта $G_{b}^{a}\left(x^{\alpha}, x^{n+a}\right)$, что $H D=\operatorname{Span}\left(\varepsilon_{a}\right)$, где $\varepsilon_{a}=\partial_{a}-\Gamma_{a}^{n} \partial_{n}-G_{a}^{b} \partial_{n+b}$.

Пусть $\nabla$-внутренняя линейная связность, определяемая горизонтальным распределением $H D$, и $N: D \rightarrow D$ - поле допустимого тензора типа $(1,1) . N$-Продолженной связностью назовем связность в векторном расслоении $(D, \pi, M)$, определяемую разложением

$$
T D=\widetilde{H D} \oplus V D
$$

где

$$
\widetilde{H D}=H D \oplus \operatorname{Span}(\boldsymbol{u}), \quad \boldsymbol{u}_{\boldsymbol{x}}=\boldsymbol{\varepsilon}-(N \boldsymbol{x})^{v}, \quad \boldsymbol{\varepsilon}=\partial_{n}, \quad \boldsymbol{x} \in D,
$$

$(N \boldsymbol{x})^{v}$ - вертикальный лифт. В базисе $\left(\varepsilon_{a}, \partial_{n}, \partial_{n+a}\right)$ поле $\boldsymbol{u}$ имеет координатное представление $\boldsymbol{u}=\partial_{n}-N_{b}^{a} x^{n+b} \partial_{n+a}$.

Допустимое тензорное поле, определяемое равенством

$$
R(\boldsymbol{x}, \boldsymbol{y}) \boldsymbol{z}=\nabla_{\boldsymbol{x}} \nabla_{\boldsymbol{y}} \boldsymbol{z}-\nabla_{\boldsymbol{y}} \nabla_{\boldsymbol{x}} \boldsymbol{z}-\nabla_{P[\boldsymbol{x}, \boldsymbol{y}]} \boldsymbol{z}-P[Q[\boldsymbol{x}, \boldsymbol{y}], \boldsymbol{z}],
$$

где $Q=1-P$, названо Вагнером (см. [6]) тензором кривизны Схоутена. Координатное представление тензора Схоутена в адаптированных координатах имеет вид

$$
R_{a b c}^{d}=2 e_{[a} \Gamma_{b] c}^{d}+2 \Gamma_{[a\|e\| \mid}^{d} \Gamma_{b] c}^{e} .
$$

Назовем тензор Схоутена тензором кривизны распределения $D$, а распределение $D$, в случае обращения в нуль тензора Схоутена,-- распределением нулевой кривизны. Частные производные 
$\partial_{n} \Gamma_{b c}^{a}=P_{b c}^{a}$ являются компонентами допустимого тензорного поля, обозначаемого в дальнейшем $P(\boldsymbol{x}, \boldsymbol{y})$.

Векторные поля

$$
\varepsilon_{a}=\partial_{a}-\Gamma_{a}^{n} \partial_{n}-\Gamma_{a c}^{b} x^{n+c} \partial_{n+b}, \quad \boldsymbol{u}=\partial_{n}-N_{b}^{a} x^{n+b} \partial_{n+a}, \partial_{n+a}
$$

задают на $D$ адаптированное поле базисов, а формы

$$
d x^{a}, \quad \Theta^{n}=d x^{a}+\Gamma_{a}^{n} d x^{a}, \quad \Theta^{n+a}=d x^{n+a}+\Gamma_{b c}^{a} x^{n+c} d x^{b}+N_{b}^{a} x^{n+b} d x^{n}
$$

- сопряженное поле кобазисов. Имеют место следующие структурные уравнения:

$$
\begin{aligned}
& {\left[\varepsilon_{a}, \boldsymbol{\varepsilon}_{b}\right]=2 \omega_{b a} \boldsymbol{u}+x^{n+d}\left(2 \omega_{b a} N_{d}^{c}+R_{b a d}^{c}\right) \partial_{n+c}} \\
& {\left[\boldsymbol{\varepsilon}_{a}, \boldsymbol{u}\right]=x^{n+d}\left(\partial_{n} \Gamma_{a d}^{c}-\nabla_{a} N_{d}^{c}\right) \partial_{n+c}} \\
& {\left[\varepsilon_{a}, \partial_{n+b}\right]=\Gamma_{a b}^{c} \partial_{n+c}} \\
& {\left[\boldsymbol{u}, \partial_{n+a}\right]=N_{a}^{c} \partial_{n+c}}
\end{aligned}
$$

Определим на многообразии $D$ римановы структуры $(D, G),(D, \tilde{G})$, полагая

$$
\begin{aligned}
& \tilde{G}\left(\boldsymbol{x}^{h}, \boldsymbol{y}^{h}\right)=\tilde{G}\left(\boldsymbol{x}^{v}, \boldsymbol{y}^{v}\right)=G\left(\boldsymbol{x}^{h}, \boldsymbol{y}^{h}\right)=G\left(\boldsymbol{x}^{v}, \boldsymbol{y}^{v}\right)=g(\boldsymbol{x}, \boldsymbol{y}), \\
& \tilde{G}\left(\boldsymbol{x}^{h}, \boldsymbol{y}^{v}\right)=\tilde{G}\left(\boldsymbol{x}^{h}, \boldsymbol{u}\right)=\tilde{G}\left(\boldsymbol{x}^{v}, \boldsymbol{u}\right)=0, \quad \tilde{G}(\boldsymbol{u}, \boldsymbol{u})=1, \\
& G\left(\boldsymbol{x}^{h}, \boldsymbol{y}^{v}\right)=G\left(\boldsymbol{x}^{h}, \partial_{n}\right)=G\left(\boldsymbol{x}^{v}, \partial_{n}\right)=0, \quad G\left(\partial_{n}, \partial_{n}\right)=1 .
\end{aligned}
$$

Предложение 2. Пусть $M$-субриманово многообразие. Тогда коэффициенты $\tilde{\Gamma}_{j k}^{i}$ связности Леви-Чивиты метрики $G$ удовлетворяют следующим соотношениям:

$$
\begin{array}{ll}
\tilde{\Gamma}_{a b}^{c}=\Gamma_{a b}^{c}, & 2 \tilde{\Gamma}_{a, n+b}^{n}=2 \tilde{\Gamma}_{n+b, a}^{n}=P_{a c}^{e} x^{n+c} g_{e b}, \\
2 \tilde{\Gamma}_{a b}^{n+c}=R_{b a d}^{c} x^{n+d}, & \tilde{\Gamma}_{n+a, n+b}^{n}=C_{a b}, \\
\tilde{\Gamma}_{a b}^{n}=\Gamma_{a b}^{n}, & \tilde{\Gamma}_{a n}^{c}=\tilde{\Gamma}_{n a}^{c}=\Gamma_{n a}^{c}, \\
2 \tilde{\Gamma}_{a, n+b}^{c}=R_{b d a}^{c} x^{n+d}, & 2 \tilde{\Gamma}_{a n}^{n+c}=-2 \tilde{\Gamma}_{n a}^{n+c}=P_{a d}^{c} x^{n+d}, \\
2 \tilde{\Gamma}_{n+a, b}^{c}=R_{a d b}^{c} x^{n+d}, & 2 \tilde{\Gamma}_{n+a, n}^{c}=2 \tilde{\Gamma}_{n, n+a}^{c}=g^{c d} P_{d b}^{e} x^{n+b} g_{a e}, \\
\tilde{\Gamma}_{a, n+b}^{n+c}=\Gamma_{a b}^{c}, & \tilde{\Gamma}_{n+a, n}^{n+b}=\tilde{\Gamma}_{n, n+a}^{n+b}=C_{a}^{b} .
\end{array}
$$

Доказательство предложения сводится к применению равенства

$$
2 \Gamma_{i j}^{m}=g^{k m}\left(A_{i} g_{j k}+A_{j} g_{i k}-A_{k} g_{i j}+\Omega_{k j}^{l} g_{l i}+\Omega_{k i}^{l} g_{l j}\right)+\Omega_{i j}^{m},
$$

где

$$
\Omega_{a b}^{n}=2 \omega_{b a}, \quad \Omega_{a b}^{n+c}=R_{b a d}^{c} x^{n+d}, \quad \Omega_{a, n+b}^{n+c}=\Gamma_{a b}^{c}, \quad \Omega_{a n}^{n+c}=\partial_{n} \Gamma_{a b}^{c} x^{n+b} .
$$

Теорема. Пусть $M$-субриманово многообразие с неинтегрируемым распределением $D$ u $\left(D, \partial_{n}, \lambda=\eta \circ \pi_{*}, G, \tilde{D}\right),\left(D, \boldsymbol{u}, \lambda=\eta \circ \pi_{*}, \tilde{G}, \tilde{D}\right)-$-ве $N$-продолженные субримановы структуры контактного типа, причем первая структура получена с помощью нулевого эндоморбизма, вторая - с помощью произвольного ненулевого эндоморфизма $N: D \rightarrow D$. Тогда соответствующие этим структурам римановы многообразия $(D, G),(D, \tilde{G})$ принадлежсат разным геодезическим классам.

Доказательство. Пусть $\nabla$ - связность Леви-Чивиты метрического тензора $G$ с коэффициентами $\tilde{\Gamma}_{j k}^{i}$ относительно базиса $\left(\varepsilon_{a}, \partial_{n}, \partial_{n+a}\right)$. Предположим противное, что $(D, G),(D, \tilde{G})$ принадлежат одному геодезическому классу. Тогда найдется такой ковектор $\psi_{i}$, что будет выполняться равенство (см. [11])

$$
\nabla_{i} \tilde{G}_{j k}=2 \psi_{i} \tilde{G}_{j k}+\psi_{j} \tilde{G}_{i k}+\psi_{k} \tilde{G}_{j i}
$$

Перепишем последнее равенство для трех разных наборов индексов. Воспользуемся тем, что $\nabla_{i} \tilde{G}_{a b}=\nabla_{i} G_{a b}$. Это равенство оказывается справедливым благодаря тому, что в базисе $\left(\varepsilon_{a}, \partial_{n}, \partial_{n+a}\right)$ выполняются соотношения

$$
\tilde{G}_{a b}=G_{a b}, \quad \tilde{G}_{n+a, n+b}=G_{n+a, n+b}, \quad \tilde{G}_{a n}=\tilde{G}_{a, n+b}=0
$$


Имеем:

$$
\nabla_{c} G_{a b}=2 \psi_{c} G_{a b}+\psi_{a} G_{b c}+\psi_{b} G_{a c}, \quad \nabla_{n+c} G_{a b}=2 \psi_{n+c} G_{a b}, \quad \nabla_{n} G_{a b}=2 \psi_{n} G_{a b} .
$$

Отсюда получаем, что $\psi_{c}=\psi_{n+c}=\psi_{n}=0$.

Пусть теперь выполняются равенства $\psi_{c}=\psi_{n+c}=\psi_{n}=0$. Вновь воспользуемся равенством

$$
\nabla_{i} \tilde{G}_{j k}=2 \psi_{i} \tilde{G}_{j k}+\psi_{j} \tilde{G}_{i k}+\psi_{k} \tilde{G}_{j i}
$$

Получаем для подходящего набора индексов следующее равенство:

$$
\nabla_{c} \tilde{G}_{a, n+b}=\nabla_{c} G_{a, n+b}-\Gamma_{c a}^{n} \tilde{G}_{n, n+b} .
$$

Мы здесь воспользовались тем, что относительно базиса $\left(\varepsilon_{a}, \partial_{n}, \partial_{n+a}\right)$ выполняется соотношение $\tilde{G}_{n, n+b}=g_{b c} N_{d}^{c} x^{n+d}$. После стандартных вычислений получаем

$$
\left(\omega_{a b}+C_{a b}\right) N_{d}^{c} x^{n+d}=0 .
$$

Отсюда при условии, что $\omega_{a b} \neq 0$, следует, что $N=0$. Теорема доказана.

\section{СПИСОК ЛИТЕРАТУРЫ}

1. Букушева А. В. Слоения на распределениях с финслеровой метрикой// Изв. Саратов. ун-та. Нов. сер. Мат. Мех. Информ. - 2014. - 14, № 3. - С. 247-251.

2. Букушева А. В. Нелинейные связности и внутренние полупульверизации на распределении с обобщенной лагранжевой метрикой// Диффер. геом. многообр. фигур. - 2015. - № 46. - С. 58-63.

3. Букушева А. В. О геометрии контактных метрических пространств с $\varphi$-связностью// Науч. вед. Белгород. гос. ун-та. Сер. Мат. Физ. - 2015. - 17 (214), № 40. - С. 20-24.

4. Букушева А. В., Галаев С. В. Связности над распределением и геодезические пульверизации// Изв. вузов. Мат. - 2013. - № 4. - С. 10-18.

5. Букушева A. В., Галаев С. В. Геометрия почти контактных гиперкэлеровых многообразий// Диффер. геом. многообр. фигур. - 2017. - № 48. - С. 32-41.

6. Вагнер B. В. Геометрия $(n-1)$-мерного неголономного многообразия в $n$-мерном пространстве// Тр. семин. по вектор. тензор. анал. - 1941. - № 5. - С. 173-255.

7. Галаев C. В. Почти контактные метрические структуры, определяемые $N$-продолженной связностью// Мат. заметки СВФУ. - 2015. - 22, № 1. - С. 25-34.

8. Галаев С. В. Почти контактные метрические пространства с $N$-связностью// Изв. Саратов. ун-та. Нов. сер. Мат. Мех. Информ. - 2015. - 15, № 3. - С. 258-263.

9. Галаев С. В. Обобщенный тензор кривизны Вагнера почти контактных метрических пространств// Чебышев. сб. - 2016. - 17, № 3 (59). - С. 53-63.

10. Галаев C. В. Допустимые гиперкомплексные структуры на распределениях сасакиевых многообразий// Изв. Саратов. ун-та. Нов. сер. Мат. Мех. Информ. - 2016. - 16, № 3. - С. 263-272.

11. Синюков Н. С. Геодезические отображения римановых пространств. - М.: Наука, 1979.

12. Bukusheva A. V., Galaev S. V. Almost contact metric structures defined by connection over distribution// Bull. Transilv. Univ. Braşov. Ser. III. Math. Inform. Phys. - 2011. — 4 (53), № 2. - P. 13-22.

Галаев Сергей Васильевич

Саратовский национальный исследовательский государственный

университет им. Н. Г. Чернышевского

E-mail: sgalaev@mail.ru 